\begin{tabular}{|l|l} 
REVISTA & $\begin{array}{l}\text { Revista Educación } \\
\text { ISSN: 0379-7082 } \\
\text { ISSN: } 2215-2644 \\
\text { revedu@ gmail.com } \\
\text { Universidad de Costa Rica } \\
\text { Costa Rica }\end{array}$
\end{tabular}

\title{
La experiencia del estudiantado mediante el uso del diario. ¿Una estrategia para la metacognición?
}

Salgado Ramírez, Alma; García Mendoza, Lorena Yazmín; Méndez-Cadena, María Esther

La experiencia del estudiantado mediante el uso del diario. ¿Una estrategia para la metacognición?

Revista Educación, vol. 44, núm. 1, 2020

Universidad de Costa Rica, Costa Rica

Disponible en: http://www.redalyc.org/articulo.oa?id=44060092040

DOI: https://doi.org/10.15517/revedu.v44i1.38291

Esta obra está bajo una Licencia Creative Commons Atribución-NoComercial-SinDerivar 3.0 Internacional. 


\section{La experiencia del estudiantado mediante el uso del diario. ¿Una estrategia para la metacognición?}

La experiencia del estudiantado mediante el uso del diario. ¿Una estrategia para la metacognición?

Alma Salgado Ramírez

Universidades Públicas e Institutos de la Secretaria de

Educación, México

lmasalgado5@hotmail.com

iD http://orcid.org/0000-0002-8302-8978

Lorena Yazmin García Mendoza

Departamento de Investigaciones Educativas (DIE-

CINVESTAV), México

l.garciam@hotmail.com

(iD http://orcid.org/0000-0002-5541-4230

María Esther Méndez-Cadena

Colegio de Postgraduados, México

mesther@colpos.mx

(iD) http://orcid.org/0000-0001-8785-0531
DOI: https://doi.org/10.15517/revedu.v44i1.38291

Redalyc: http://www.redalyc.org/articulo.oa?id=44060092040

Recepción: 24 Julio 2019

Aprobación: 17 Diciembre 2019

\section{RESUMEN:}

El diario visto como escritura reflexiva puede ayudar al profesorado a establecer un canal de comunicación directo y cercano con el estudiantado. El propósito del presente texto es fundamentar cómo el uso del diario puede permitir a cada estudiante el desarrollo de habilidades de metacognición. Desde una metodología cualitativa y empleando el software Atlas Ti, se estudiaron 82 diarios de clase de estudiantes de posgrado en educación y especialidad en docencia universitaria de dos instituciones de educación superior, para dar cuenta de su experiencia a partir de lo que perciben y relatan en su diario. Los resultados revelan que el uso del diario genera reflexión en el estudiantado y desarrollo de diferentes habilidades, que contribuyen a la metacognición y toma de conciencia en el aprendizaje. También se observa que el diario permite al cuerpo docente identificar pautas para mejorar su desempeño con acciones que coadyuven a enfrentar los desafíos de la educación superior en el siglo XXI.

Palabras clave: Diario de aprendizaje, Metacognición, Educación superior, Proceso de aprendizaje.

\section{Abstract:}

A diary is perceived as reflective writing that can help teachers establish a direct communication channel with students. The purpose of this article is to substantiate that daily journal writing helps facilitate the development of student metacognition skills. Using a qualitative methodology with Atlas Ti software, a group of 82 postgraduate Education Majors from two universities shared their experiences with regards to journal writing. The results reveal that keeping a diary stirs thought among students, leading to the development of skills that contribute to metacognition and greater awareness about learning. We also observed that journal writing helps teachers identify guidelines for improving their performance with actions that will assist them in facing the challenges of Higher Education in this XXIst century.

KEYWORDS: Learning Journal, Metacognition, Higher Education, Learning Process. 
y que sirve para recuperar el elixir de la experiencia

Fuente: Christopher Vogler

Writing is a risky inward journey

used to verify the depth of one's soul

and help recover the elixir of experience

Fuente: Christopher Vogler

\section{INTRODUCCIÓN}

Cada docente cuenta con diversos recursos para ayudar a sus estudiantes a reflexionar sobre su proceso de aprendizaje, entre ellos se encuentra el diario. Para esta investigación el diario es considerado un hipomnemata, utilizando el término empleado por Foucault para dar cuenta de "una recopilación de cosas leídas, y oídas y soporte de los ejercicios de pensamiento [...] por la apropiación, la unificación y la subjetivación de un ya-dicho fragmentario y elegido - en la escritura de sí” (Didi-Huberman, 2013, p.21).

Este tipo de escritura se caracteriza por ser personal y constituye un material que funciona como marco para la elaboración de ejercicios frecuentes como leer, releer, reformular ideas; en este sentido se aleja de la idea de un diario íntimo que sirve para confesarse o para relatar lo oculto, por ello se acerca más a la condición de un diario de trabajo, que permite al autor reunir lo que ha experimentado a través de lo visto, lo escuchado, lo leído, con el fin de comprender su experiencia de pensamiento.

La escritura del diario implica una reorganización y recomposición de lo que se ha vivido, a la vez que supone un posicionamiento, pues para saber dice Didi-Huberman "hay que tomar posición" (2013, p.9); hay que situarse en el tiempo y en el lugar desde el cual uno está para afrontar algo, pero también requiere posición para ubicar los deseos, lo que se quiere lograr, hacia dónde se quiere ir y lo que se está dispuesto a renunciar.

En la práctica docente existen diversas herramientas las cuales pueden ser utilizadas para apoyar el proceso de aprendizaje del estudiantado. El diario de cada estudiante es una de ellas. En él, plasman su experiencia de aprendizaje, reflexionan sobre lo que hicieron, escriben con sus propias palabras acerca de lo que vivieron y cómo lo vivieron. La fuerza de esto, reside principalmente en lo que dice Giraldo (2015) “[...] la escritura no es un fin en sí mismo. Es un medio para aprender o para dar sentido a la existencia. Porque escribir es una manera de existir, de construir y reconstruir el mundo propio" (p. 42), es decir, un medio para que puedan darle sentido a su proceso de enseñanza y aprendizaje.

Para Paniagua y Palos (2009) "Escribir es una conquista humana, un acto de cognición. Escribir sobre lo que se hace es un acto de pensamiento superior y, en términos docentes, un camino para regresar sobre nuestros pasos y aprender de ellos" (p.13). En este sentido, uno de los retos de la educación superior del siglo XXI es propiciar espacios de aprendizaje que lleven al estudiantado a adquirir y desarrollar habilidades cognitivas y afectivas de orden superior, que les permitan enfrentarse a las transformaciones sociales, económicas y ambientales de la sociedad actual.

El diario es un género de escritura que favorece la introspección, el diálogo con uno mismo; permite reflexionar sobre lo que resultó significativo, poner en evidencia la subjetividad de la experiencia y recuperar a través de lo escrito aquello que aconteció para valorarlo, meditarlo y evaluarlo. Según Jurado (2011) el diario puede ser visto "como un instrumento o herramienta de (investigación, de autoformación, de evaluación, etc.) que nos ayuda a configurar y recrear nuestros pensamientos, sentimientos y sobre todo nuestra palabra y nuestra acción” (pp.174-175).

Históricamente los diarios han servido para mostrar algo de las relaciones que el autor establece consigo mismo y con su mundo. Un ejemplo lo constituyen los cuadernos de apuntes que Hannah Arendt escribió 
entre 1950 y 1973 y que posteriormente dieron paso a su Diario filosófico; así como las anotaciones y reflexiones que Bertolt Brecht redactó durante su exilio y componen su Diario de trabajo. Estos textos son un ejemplo de escrituras de orden personal que permitieron a sus autores comprender mejor su experiencia y la lógica de su época.

No obstante, en el repertorio de estrategias que emplea el profesorado para apoyar a sus estudiantes en su proceso de aprendizaje la presencia del diario ha sido marginal; los métodos y técnicas de enseñanza que se han consolidado como predominantes en la educación superior están referidas mayormente a la promoción de la comprensión y estas operan mediante la organización de la información a través de instrumentos como organizadores visuales y gráficos. La preponderancia que lo cognitivo ha tenido en la concepción del aprendizaje, sugiere que lo más importante a lograr por el estudiantado es el manejo de la información y el dominio de los contenidos. Ante esta situación, Roig y Cobos (2019), proponen que es "necesario tener en cuenta modelos didácticos que permitan potenciar las capacidades de los y las estudiantes" (p.129), tales como la actividad reflexiva, el reconocimiento del error, la identificación del problema y el planteamiento de soluciones (García, 2006, González, Cárdenas y Arellano, 2017), en suma todo lo relacionado con los procesos metacognitivos y la expresión de las emociones.

En este marco, la presente investigación tiene como objetivo fundamentar la experiencia del uso del diario como recurso de doble beneficio, pues por un lado le permite al estudiante darse cuenta de cómo aprende y por otro, al docente le ofrece elementos para mejorar e innovar en su práctica educativa desde una mirada crítica.

El texto está organizado en cuatro apartados. En la primera parte se exponen los fundamentos teóricos sobre el uso del diario por parte de cada estudiante. Posteriormente, se presenta el análisis de las narrativas presentadas en los diarios. En un tercer apartado se muestran los hallazgos de la investigación apoyados por los referentes teóricos que guían esta investigación y, finalmente se concluye con algunas reflexiones y recomendaciones.

\section{Marco TEÓRICO}

La literatura especializada en el área utiliza diferentes términos para identificar al diario. Se define al diario como un recurso pedagógico el cual le permite al estudiantado y al profesorado reflexionar sobre lo sucedido en el aula a través de narrar sus experiencias en ella y en algunos casos también fuera de ella. Para el caso de esta investigación el diario es considerado un hipomnemata, constituye una memoria material a partir de la cual el alumnado tiene acceso, a través de su propia escritura, a lo que leyeron, escucharon, pensaron; el diario compone la materia prima mediante la cual podrán releer, repensar para comprender mejor su experiencia de pensamiento y la construcción de sus ideas.

Para autores como Messina (2019) el diario es un tipo de texto que celebra la autonomía y recupera la escritura de la experiencia. En este mismo orden de ideas Jurado (2011) señala que un rasgo importante del diario es que deja "constancia de la experiencia y de la vivencia de aquellas personas que lo escriben. Así como la posibilidad de poner en común esas experiencias y contrastarlas, para hacer más comprensible todo el proceso seguido" (p. 176).

En este sentido, la escritura se convierte en la posibilidad de recuperar la experiencia, pero para hacerlo es necesario habilitar un espacio, como expone Larrosa (2018) "retirarse a escribir es establecer una separación que une, una distancia que aproxima. El escritor se separa de la realidad, de la vida y de los otros para recuperarlos, en el alejamiento, de otra manera." (p.1). Entonces, recuperar la experiencia a través de la escritura, supone hacer una pausa y establecer una distancia que permita volver a nosotros mismos, formulando preguntas, generando un diálogo con lo que se vive y sucede, pero que no se logró apreciar con detenimiento debido a la presión y el acelere de la vida diaria. 
Diversos autores coinciden en señalar, que la escritura en la clase, en el nivel superior ha estado estrechamente vinculada a la exigencia, al cumplimiento; escribir se convierte en una evidencia, en un producto esperado, lo que hace que su valor se pierda y termine como una mercancía. Por ello, recuperar la escritura a través del diario puede abrir un espacio para que cada estudiante asuma una distancia que le ayude a encontrar el misterio de lo cotidiano o desnaturalizar aquello que ya no se cuestiona, como vuelve a decir Larrosa (2018)

[...] cuando intentamos escribir lo que creíamos saber empieza a parecernos misterioso, lo que creíamos conocer nos parece desconocido y enigmático, lo que creíamos simple nos parece tan complejo que casi es inapresable. Como si el simple hecho de comenzar a escribir tuviera como efecto la desfamiliarización de lo que nos parecía familiar, la desnaturalización de lo que nos parecía natural, la extrañeza de lo que nos parecía trivial y poco significativo (p.3).

En efecto, el diario es un tipo de escritura que ayuda a recomponer la experiencia es "una máquina de dejar huellas" (Ojeda, 2017, parr.5). Huellas que son de interés para esta investigación.

\subsection{Tipos de diario}

Como cualquier documento personal, el diario puede utilizarse para diversas y variadas finalidades. Existen diarios personales, escolares, de viaje, de campo, del docente. No obstante, su esencia, como se había adelantado, se encuentra en la importancia que concede al elemento subjetivo o interno en su conexión con lo objetivo o externo. Sicilia (1999), señala que "dentro del ámbito educativo, el diario de cada estudiante es especialmente interesante cuando se quiere priorizar la visión o representación subjetiva que el estudiantado tiene en general del contexto educativo que le rodea" (p. 27) y su proceso de aprendizaje.

Ospina (2016) en su texto El diario como estrategia didáctica presenta una clasificación que incluye el diario personal o diario de vida; diario de viaje; diario escolar; diario como archivo de experiencias o método para documentar vivencia; diario de campo y diario docente.

En la modalidad de diario escolar, lo que se registra es el trabajo del alumnado, su producción e interacción en clase, a la vez que da cuenta de sus reflexiones, preguntas, respuestas. Un ejemplo de este tipo de diario es el que comparte el estudio de Carlino (2003) que utilizó diarios de clase con estudiantes de postgrado para retroalimentar entre pares el trabajo de tesis.

El diario como archivo de experiencias o método para documentar vivencia, es un tipo de diario ampliamente usado en los trabajos de investigación, pues lo que se busca es ejercitar la reflexión a partir de lo que acontece en la vida cotidiana; documentar y plasmar por escrito la experiencia y la reflexión que se generó a partir de ella es su propósito. El estudio desarrollado por Monsalve y Pérez (2012), muestra cómo los textos escritos por docentes constituyen el insumo para identificar y analizar buenas prácticas.

Para el caso del diario docente según Ospina (2016) permite recoger "observaciones de hechos considerados relevantes para el y la docente y que contribuyen al conocimiento de la realidad del grupo de estudiantes, así como plasmar y reflexionar críticamente su propia actividad teórico-práctica” (parr.10). El estudio elaborado por Vain (2003) emplea los diarios académicos como una estrategia para la formación reflexiva del cuerpo docente y a través de su uso busca articular la teoría y la práctica para la formación de profesionales reflexivos. Este autor propone el uso del diario académico como una manera de cuestionar el predominio de la racionalidad técnica en la enseñanza superior y de postgrado.

\subsection{Ventajas de uso}

Las ventajas que reporta el uso del diario son múltiples, entre ellas se pueden mencionar la capacidad de representar lo sucedido en una experiencia de aprendizaje concreta por el mismo estudiantado (Porlán y Martín, 2000). Esto, lo conduce a un trabajo de metacognición que le ayuda a reconocer cómo está 
aprendiendo, así como las dificultades a las cuales se enfrenta durante el proceso. Al docente por su parte le permite verificar lo relacionado con el desarrollo de habilidades de orden superior tales como sintetizar, evaluar, crear, hacer razonamientos creativos y evaluar lo que están logrando sus estudiantes.

Báez y Otero (2013) muestran que los aportes que comporta el uso del diario están referidos al desarrollo de la autonomía, a la capacidad de aprender a aprender y en particular al desarrollo de la escritura.

El diario de aprendizaje también es útil para el profesorado y la persona investigadora, dado que puede servir como fuente de información sobre los procesos de autoobservación, autocrítica y autoevaluación del estudiantado y, además, le ayuda a obtener información sobre aspectos de los aprendientes tan diversos como, por ejemplo, los factores personales, los aspectos afectivos, socioculturales, cognitivos o estratégicos, sus necesidades de aprendizaje, etc.

Otra de las ventajas, es la retroalimentación que cada docente le proporciona al alumno. De esta manera el estudiantado puede ir contrastando su propia visión con la visión docente e ir haciendo énfasis en sus fortalezas y áreas de oportunidad. Con la retroalimentación dada, tiene un parámetro más certero sobre las áreas a mejorar a través de diferentes estrategias que el mismo profesorado le va señalando o que incorpora durante su proceso metacognitivo.

Además, es una manera de acompañar, como lo mencionan Cebrián, Pérez y Cebrián (2017), “[...] no los dejemos solos reconstruyendo la narrativa de sus pensamientos y experiencias prácticas" (p.3), sino que acompañados van dando cuenta cómo aprenden o qué se les dificulta. Dicho de otra manera, se establece un proceso comunicativo entre el profesorado y el alumnado.

Otra de las ventajas que se pueden añadir es la que mencionan López y Roger (2014) sobre la posibilidad de enriquecer la evaluación con los datos que arrojan los diarios del y la estudiante. El cuerpo docente cuenta con un recurso más para investigar que le ayudará a obtener pistas para la mejora de su práctica docente. Darse cuenta qué estrategias no favorecen el proceso de aprendizaje y cuáles podría incorporar para potenciar el desarrollo de las habilidades que cada estudiante necesita.

Por último, cabe mencionar que esta herramienta va en concordancia con aquellas metodologías de enseñanza y modelos de aprendizaje centrados en el alumnado que permiten alejarse del énfasis de la figura docente como protagonista del proceso educativo y dar paso a una relación de enseñanza más armónica entre docentes y estudiantes (López y Pérez, 2018).

\subsection{Enseñanza - educación superior}

Uno de los retos que enfrenta la educación superior es ir más allá de solo centrarse en el desarrollo de habilidades, para conducir al estudiantado hacia procesos de reflexión más complejos que recuperen lo subjetivo y desarrollar habilidades de orden superior que mejoren su proceso de aprendizaje y de vida. Por ello, como se expuso anteriormente, cobra mayor relevancia la selección e implementación de herramientas que permitan la sincronía entre enseñar y aprender.

Asimismo, algunas de las investigaciones que relatan el uso del diario muestran los beneficios de usarlo como una estrategia pedagógica. El caso de Hortigüela (2017) confirma que el usar técnicas narrativas con énfasis en la reflexión (diario) con técnicas de evaluación mejoró la capacidad de metacognición.

Otras investigaciones como la de Porlan y Martín (2000) y González (2018) manifiestan que el diario favorece la capacidad de representar lo sucedido en una experiencia de aprendizaje concreta por el mismo estudiante, así como fortalecer la escritura académica que es vital para la producción científica. Por su parte, Jiménez, Rossi y Gaitán (2017) demuestran que el uso del diario epistémico, ayuda a la reflexión del y la docente sobre su propia práctica.

Autores como González, Vargas, Del Campo y Méndez (2017) en su investigación concluyen que las y los estudiantes 
[...] cuentan con pocas herramientas para ser capaces de organizar, clasificar, interpretar, representar y explorar los nuevos conocimientos y así integrarlos con lo que ya conocían, logrando con ello un aprendizaje significativo; condición fundamental para llegar a ser capaces de pensar por sí mismos, construir conocimiento, lograr autonomía en el aprendizaje y desarrollar el pensamiento crítico (p.85).

El diario de estudiante puede ser una de ellas. En suma, esta herramienta también contribuye a que el estudiantado se vaya encaminando hacia el desarrollo de habilidades para la investigación fundamentales en su formación universitaria.

\section{Metodología}

La metodología utilizada en la presente investigación es de tipo cualitativo con corte descriptivo interpretativo, al observar como el diario genera procesos de metacognición, desde una mirada de comprensión a lo acontecido. Los participantes en el estudio fueron estudiantes de segundo semestre de la maestría en Diseño Estratégico e Innovación y del tercer módulo de la Especialidad en Docencia Universitaria, ambos programas se encuentran inscritos en universidades mexicanas. La muestra del estudio fue por conveniencia de acuerdo a los intereses de la investigación, como lo mencionan Gay y Airasian (1996) “ellos pueden proveer información pertinente acerca del tema específico a investigar" (p.209).

Se analizaron un total de 82 diarios de clase de un total de 646 fragmentos. Para la construcción de los diarios se solicitó que cada estudiante escribiera los aprendizajes cognitivos y afectivos alcanzados en cada sesión, se plantearon preguntas detonadoras para propiciar la escritura tales como: ¿qué es lo más significativo de la sesión?, ¿qué no deberías olvidar de la sesión?, ¿cómo pondrías en práctica lo aprendido?, esto al final de cada sesión a lo largo de un cuatrimestre. Los participantes en la investigación compartían el ser profesionistas de distintas disciplinas y se desempeñaban como docentes o tenían la aspiración de serlo. Todos dieron su consentimiento y autorización para que sus diarios fueran utilizados en la presente investigación.

El envío de los diarios se hizo a través de Moodle y Google Docs; las opciones tecnológicas se fueron ampliando a solicitud de cada estudiante, quienes propusieron el uso de Facebook, Dropbox y correo electrónico, lo cual favoreció la retroalimentación por parte del personal docente y la incorporación de la tecnología en el aula.

Para el análisis de los diarios se utilizó el software Atlas Ti, el cual permitió identificar categorías a través del método de Comparación Constante, comúnmente utilizado en la metodología cualitativa. Este método consiste en encontrar en los datos recolectados unidades de información, las cuales se agrupan en categorías que son ponderadas de acuerdo al número de menciones o profundidad de las aportaciones; estas categorías se contrastan con los elementos teóricos de la investigación y así se llega a la teorización de los hallazgos (Martínez, 2013).

Inicialmente se definieron 42 códigos de información, a partir de los cuales se organizaron nueve categorías: Aprendizaje; Recursos de aprendizaje; Método de enseñanza; Aprendizaje emocional; Dificultades de aprendizaje; Desarrollo de habilidades; Logros; Expectativas y Contexto del estudiantado. En este documento se da cuenta de seis categorías, por ser las de mayor énfasis en el discurso y testimonio del alumnado. No obstante, se planea en un segundo momento continuar con la investigación abordando las categorías excluidas en este documento. Las categorías finales son: Trabajo docente; Aprender jugando; Metacognición; Trabajo colaborativo; Emociones y Aprendizaje significativo. En la Tabla 1, Categorías de análisis, se describen cada una. 
TABLA 1

Categorías de análisis

\begin{tabular}{|c|c|}
\hline Categoria & Descripción \\
\hline Metacognición & $\begin{array}{l}\text { En este apartado se da cuenta de los procesos } \\
\text { de cambio, reflexión, interrogación, } \\
\text { descubrimiento y conocimiento de si mismo de } \\
\text { los participantes. Y se valora la capacidad de } \\
\text { aprender a aprender. }\end{array}$ \\
\hline $\begin{array}{l}\text { Aprendizaje } \\
\text { Significativo }\end{array}$ & $\begin{array}{l}\text { Esta categoria describe lo significativo del } \\
\text { aprendizaje, los sentidos que dan estudiantes } \\
\text { sobre lo que aprenden, incorporando nuevas } \\
\text { estructuras a sus conocimientos previos y } \\
\text { encontrando utilidad para todos los ámbitos de } \\
\text { su vida cotidiana. }\end{array}$ \\
\hline Emociones & $\begin{array}{l}\text { Muestra las emociones que expresan } \\
\text { estudiantes tales como sus expectativas, el } \\
\text { clima de la clase, su disposición para aprender } \\
\text { y los logros obtenidos, asi como la incidencia de } \\
\text { las actividades cotidianas y laborales en su } \\
\text { aprendizaje. También se aprecia el } \\
\text { agradecimiento al docente por los aprendizajes } \\
\text { logrados. }\end{array}$ \\
\hline $\begin{array}{l}\text { Trabajo } \\
\text { docente }\end{array}$ & $\begin{array}{l}\text { Describe los testimonios de estudiantado sobre } \\
\text { la figura del facilitador del aprendizaje } \\
\text { identificando enfoques, estilos, modo de } \\
\text { organización de la clase, tipo de actividades } \\
\text { efectuadas, así como el grado de vinculación y } \\
\text { empatía con el profesorado y el nivel de } \\
\text { satisfacción de cada estudiante. }\end{array}$ \\
\hline $\begin{array}{l}\text { Trabajo } \\
\text { Colaborativo }\end{array}$ & $\begin{array}{l}\text { En esta categoria se expone la percepción de las } \\
\text { y los estudiantes ante el trabajo colaborativo, el } \\
\text { tipo de relaciones que establecen entre ellos y, } \\
\text { las dificultades y demandas que representan el } \\
\text { trabajo con otros. Asi como las emociones que } \\
\text { se producen y manifiestan cuando llegan a la } \\
\text { meta propuesta. }\end{array}$ \\
\hline $\begin{array}{l}\text { Aprender } \\
\text { Jugando }\end{array}$ & $\begin{array}{l}\text { Esta categoria detalla la experiencia del } \\
\text { aprendizaje como algo divertido. Establece la } \\
\text { relación de gozo y disposición con la } \\
\text { incorporación de nuevos conocimientos. Jugar } \\
\text { facilita la apropiación de temas o contenidos } \\
\text { que pueden ser densos o considerados tediosos. }\end{array}$ \\
\hline
\end{tabular}

Fuente: Elaboración propia.

La relevancia de estas categorías a la luz de identificar cómo el diario le permite al estudiantado darse cuenta de cómo aprende y al docente establecer acciones de cambio para mejorar, lo cual se presenta a continuación.

\section{ANÁLISIS Y DISCUSIÓN DE RESULTADOS}

Cada una de las categorías identificadas en el análisis da cuenta del sentir y la interpretación que el estudiantado hace respecto a la dimensión referida. A continuación, se presenta el análisis y discusión de cada una de ellas. 


\subsection{Metacognición}

Darse cuenta de cómo se aprende, qué estrategias ayudan a construir conocimiento y qué dificultades se afrontan es una habilidad que hay que desarrollar durante el proceso educativo. Esta tiene que ver con el cada vez más conocido y valorado constructo llamado metacognición. Según Cerchiaro, Paba y Sánchez (2011):

La metacognición puede definirse como el grado de conciencia o conocimiento de los individuos sobre sus formas de pensar (procesos y eventos cognitivos), sus contenidos (estructuras) y la habilidad para controlar esos procesos con el fin de organizarlos, revisarlos y modificarlos en función de los progresos y los resultados del aprendizaje (p.101)

$\mathrm{Al}$ analizar, los discursos de estudiantes, resaltan de manera muy significativa y repetitiva, frases como: darme cuenta; ha cambiado mi forma de pensar; de reflexionar; me senté a pensar; aprender de lo aprendido, lo cual evidencia aspectos relacionados con la metacognición. Describen sus momentos de reflexión, transformación de sus estructuras mentales, dificultades para construir el conocimiento, así como la valoración de sus emociones y sentimientos.

Los siguientes extractos son evidencia de ese proceso reflexivo:

Esta frase me hizo reflexionar sobre cómo muchas veces no estamos preparados para vivir ciertas experiencias, pero cuando es tu momento simplemente llega (C. L., comunicación personal, octubre 2016).

Afectivamente conforme pasan las semanas las emociones son orientadas a desarrollar las actividades encomendadas de manera más rápida y no se manifiestan tanto a flor de piel como al inicio de la maestría (M. F., comunicación personal, noviembre 2016).

Revelan también las dificultades y dudas que se presentan en el momento de aprender, cómo:

Al empezar a leer y empaparme más de este tema han ido surgiendo pensamientos de duda y al mismo tiempo surge la necesidad de saber y conocer más para entender toda esta evolución (G.R., comunicación personal, junio 2016).

El desarrollo de la metacognición no se da en el mismo nivel para todos los alumnos, en algunos el proceso es más elaborado y se asocia en la significancia que cada participante da al trabajo en el aula, como puede constatarse en el siguiente fragmento:

\section{El aprender me hace más consciente.}

El aprender me hace conocer algo de mi que no conocía.

El aprender me hace mejor persona. El aprender es un reto que me hace sentir en 'tensión', Es interesante. En esta materia vamos a desarrollar habilidades como: leer, escribir, escuchar, observar, hablar, deducir, construir, crear, reflexionar (A.C., comunicación personal, noviembre 2016).

El uso del diario permite evidenciar cómo se experimenta la metacognición y ratifica la ventaja de su uso señalado por Báez y Otero (2013) al contribuir en la capacidad de aprender a aprender y a la autoevaluación, mismos que implican procesos de gran elaboración que se escapan a otras herramientas didácticas tradicionales.

\subsection{Aprendizaje significativo}

La mayoría de estudiantes describen en sus diarios que su aprendizaje es significativo, es decir, que lo que están aprendiendo tiene que ver con su vida cotidiana, lo resignifican ellos mismos y lo incorporan a su estructura cognitiva y afectiva. Esto se produce, ya sea trabajando de manera individual o en equipo. Moreira (2012) explica que aprendizaje significativo es aquel que conecta los conocimientos previos que posee cada estudiante con el nuevo contenido. De esta manera se adquieren nuevos significados o una "nueva estabilidad cognitiva" (p. 30). Algunos estudiantes describen esta vivencia así:

En cuanto al portafolio de evidencias el aprendizaje resultó significativo para mí, ya que lo llevé a la práctica por medio del ejercicio práctico de un portafolio de evidencias para el trabajo en equipo realizado en mi clase (M.C., comunicación personal, julio 2016). 
Esta materia se ha convertido en la mejor materia que hemos llevado hasta el dia de hoy. Esta materia es el "parte aguas"

1 no solo de la especialidad que estamos cursando, sino también de toda la vida en la docencia (O. R., comunicación personal, junio 2016).

Varios fragmentos del diario evidencian ese cambio en su estructura cognitiva resultado de aprender algo útil y que pueden relacionar y aplicar en otros contextos con los que interactúan.

Me doy cuenta y me sorprendo de lo mucho que hemos aprendido y que realmente tiene aplicación no solo para un proyecto de investigación, sino que las habilidades de observación e investigación se aplican a la vida diaria (R.M., comunicación personal, noviembre 2016).

Hoy me siento más interesado en el tema, ya que poco a poco, voy entendiendo su relación con el diseño estratégico, y el papel que juega (M. S., comunicación personal, diciembre 2016).

Lo más significativo [...] saber que el proceso del aprendizaje se consideran los elementos cognitivos, los procesos de atención, selección, memorización y las capacidades de razonamiento, para desarrollar la competencia de aprender a aprender (J.R., comunicación personal, diciembre 2017).

También se encuentran testimonios de estudiantes quienes declaran que ese proceso de construcción del conocimiento, es facilitado en parte, por el andamiaje que le brinda la figura docente. Conviene subrayar, que en las metodologías activas donde el alumnado es el centro del proceso de aprendizaje, la figura del facilitador es esencial para favorecer ese ambiente, en particular el tipo de canal de comunicación que establece y la retroalimentación que le ofrece al estudiantado, como se puede apreciar en los siguientes testimonios:

En el caso de la pronta retroalimentación a las tareas he podido percatarme de la retroalimentación como momento fundamental (C.Z., comunicación personal, junio 2016).

Las indicaciones que nos han dado en cuanto al modo y la forma en cómo hemos estado trabajando (M.G., comunicación personal, junio 2016).

Me di cuenta que es importante permanecer asesorada para no desviarme en este momento con la ayuda de mis profesores (L. R., comunicación personal, noviembre 2017).

A través de los diversos testimonios se confirma, que el aprendizaje significativo según Rodríguez (2014) es, "tanto en el proceso como el producto final del mismo" (p.4), un alumno lo expresa así:

Creo que más allá de lo que hemos visto en clase, hablábamos de desarrollar habilidades, se trataba de hacerlas parte de ti y de tu vida diaria. En ese sentido siento que he hecho un enorme cambio no solo en observarlos a todos, sino en aprender de mi también (P. T., comunicación personal, octubre 2017).

Por tanto, el diario, como señalan Cebrián et al. (2017), es un medio que permite al estudiante darse cuenta de cómo aprende lo que repercute en un mejor aprendizaje.

\subsection{Emociones}

Para autores como Ausubel (2002), Goleman (1997), Mayer, Caruso y Salovey (1999) la emoción abre la puerta al proceso de aprendizaje. Un elemento fundamental en cualquier proceso educativo es la construcción de una relación que esté mediada por el interés, mismo que pondrá en evidencia la articulación entre razón y emoción, cuestión fundamental para la interacción entre los sujetos, objetos y contextos.

En el análisis desarrollado se observó que los participantes ponen de manifiesto una diversidad de emociones; algunas revelan sus expectativas del curso, el clima de la clase y el grupo; otras exponen sus preferencias en torno a los temas y actividades que son de su interés; unas más corresponden a cómo viven el proceso de aprendizaje, los logros obtenidos; y otras tienen que ver con sus actividades cotidianas, laborales y profesionales y, con el reconocimiento que hacen a la tarea ejecutada por el profesorado.

Así, por ejemplo, los estados de ánimo que corresponden a la disposición hacia el aprendizaje suelen expresarse en frases como: 
[me siento] animado a hacer las cosas y seguir adelante en la buisqueda de lograr un ritmo más rápido de trabajo (C. G., comunicación personal, julio 2016).

Me siento emocionado, con ganas de inventar, de seguir creando (E. C., comunicación personal, diciembre 2016).

Estas menciones dan cuenta de cómo el estado de ánimo guarda relación con la productividad estudiantil. Elizondo, Rodríguez y Rodríguez (2018) indican que los estados emocionales positivos fortalecen la sinapsis y aumentan la eficiencia del proceso cognitivo.

Otras manifestaciones sobre las emociones y los sentimientos aparecen cuando los participantes escriben sobre cómo se sintieron al inicio del curso; las expresiones más recurrentes son:

Me agradó el grupo, se siente buena vibra (H. M., comunicación personal, octubre 2017).

Debo confesar que me sentí algo nerviosa y entusiasmada (O. B., comunicación personal, junio 2016).

Me sentía algo nervioso, pero a la vez más seguro de haber tomado la decisión de entrar a este postgrado (P.D., comunicación personal, diciembre 2017).

Hasta que tuvimos la primera clase, tenía cierta sensación de temor (D. S., comunicación personal, junio 2016).

Este tipo de frases también se repiten cuando comparten sus experiencias sobre su participación en el grupo y el clima de la clase:

Me sentí nerviosa pues se me dificulta convivir con personas a las que no conozco (R. T., comunicación personal, octubre 2017).

Estaba un poco nerviosa ya que la actividad que habíamos planeado necesitaba de la participación de las personas, por lo que no estaba muy segura de que iba a funcionar (J. M., comunicación personal, noviembre 2017).

El hecho de sentirse parte del grupo, de integrarse influye en las actitudes de los participantes, por tanto, el diario además de facilitar la expresión ofrece un espacio para que a través de la escritura se vayan liberando y reconociendo las emociones que podrían convertirse en un obstáculo para el aprendizaje.

Los participantes también expresan sus emociones y sentimientos cuando hablan sobre los resultados de las actividades efectuadas. Palabras como nervioso, estrés; presión son recurrentes en los relatos de estudiantes, en particular cuando narran sus impresiones sobre cómo avanza la tarea que ejecutan o cuando comentan sobre las decisiones que tomaron. Pero también se encuentran expresiones como:

Abrí mi mente, me relajé y todo me fue más fácil (M. L., comunicación personal, octubre 2017).

Fue interesante, fue agradable tener este tipo de ejercicio (I. L., comunicación personal, diciembre 2017).

Al hacer este ejercicio me sentí bastante a gusto (A. C., comunicación personal, octubre 2017).

Estos testimonios permiten reconocer que el aprendizaje no es afectivamente neutral, ni tampoco puro, en él hay mixturas que se activarán y predominarán dependiendo de las acciones y situaciones a las que estén expuestos los participantes, por ello el trabajo que desarrolle el personal docente es fundamental.

En los diarios se puede apreciar que el alumnado también expone cómo se siente en su día fuera de la clase, cómo ha sido su semana de trabajo y la valora emocionalmente en relación con su desempeño académico. Tal y como lo señalan varios autores (Delors, 1996; Sánchez, 2015), la construcción de conocimiento supone un ambiente de confianza, un clima armónico, de mutuo apoyo que ayuda a que los adultos se relacionen positivamente con su proceso de adquisición de conocimiento.

En un número menor se encuentran testimonios de participantes que hablan de lo que movilizó en ellos alguna participación o comentarios de sus compañeros:

En especial el tema de mi compañera me removió muchos sentimientos al ver su tema de investigación (A. S., comunicación personal, octubre 2016).

Conviene subrayar que la voz del estudiantado puede propiciar un cambio de mirada, como lo afirma García (2018) "una mirada decidida, atenta, posicionada puede servir para enfrentar los pesos de estos tiempos e imaginar otros mundos posibles” (p. 114). 
El reconocer y conocer las emociones es un elemento presente en el aprendizaje, en el bienestar y desarrollo de las personas (López, 2005), por lo que el hecho de que en los diarios sean mostradas de forma clara, muestra la relevancia que tienen las emociones en el proceso formativo y cómo el diario puede servir para que sean socializadas.

\subsection{Trabajo del profesorado}

En los testimonios de cada participante se observa una fuerte referencia al trabajo docente, a la forma de trabajar, de dar las clases, a su estilo. La escritura de los diarios revela la experiencia que tienen como estudiantes -que va desde primaria hasta universidad-. Algunas asociaciones que se identifican en los diarios y que resultan de interés por evidenciar la forma en que se conceptualiza el trabajo docente son:

- Relacionado con enfoques, perspectivas y metodologías de enseñanza-aprendizaje.

- Vinculado a formas de hacer, organizar y secuenciar, tanto actividades como ejercicios.

- Asociado a las formas en que el y la docente ayuda y propicia el construir conocimiento.

- Ligado a lo que produce su actuación, con una fuerte orientación en aquello que les permitió cambiar su vida y su perspectiva.

- Vinculado al nivel de satisfacción, que va desde considerar la clase divertida, entretenida hasta interesante y atractiva.

- Visto como autorreflexión sobre estilo y preferencias de aprendizaje, en particular sobre cómo les gusta aprender o lo que les ayuda a mantener su atención y no perder la concentración e interés.

- Relacionado con las expectativas sobre mantener y sostener la metodología de trabajo durante todo el curso y que sea semejante en todas las asignaturas que cursan.

o anterior muestra que cuando se habla de trabajo docente hay distintas maneras de vivirlo y acciones diversas a través de las cuales se identifica; se hace referencia a la actuación del cuerpo docente, pero también a un conjunto de relaciones, producciones, efectos que pueden ser considerados por el y la docente para repensar su práctica y mejorarla. Los siguientes testimonios dan cuenta de ello:

Las clases fueron siempre una fuente de inspiración para trabajary además de que con las dinámicas iniciales [...] nos desconectaba de las preocupaciones que traíamos cargando de la semana y en particular me hacia sentir bien conmigo mismo que es algo que en la rutina suele perderse (M.B., comunicación personal, diciembre 2016).

Esta materia, [...] ha logrado que todos nos mostremos como humanos que somos, las actividades que realizamos nos permiten reflejar lo que sentimos, nuestras formas de ver el mundo y sobre todo mostrarnos humildes y esto hace muchisima coherencia con los valores que se definieron en la primera sesión (M.M., comunicación personal, diciembre 2016).

La referencia al trabajo del y la docente en los diarios no solo pone a la vista que su actuación es un medio de aprendizaje para las y los estudiantes, sino también muestra un camino para que el y la docente identifique la diversidad de aspectos que entran en juego en el aprendizaje de las y los estudiantes. Parga (2007) señala que "La educación para la comprensión genuina y el desarrollo del pensamiento constituye una empresa difícil y de alguna manera técnica, que requiere teorías y herramientas para la enseñanza y el aprendizaje que estén a la altura del reto" (p.10); por ello cuando en la educación el centro es el y la estudiante esta mejora la profundidad de su aprendizaje. Al respecto sirven un par de testimonios

Este tipo de enseñanza hace que tenga mayor interés en aprender y cambia la visión de la monotonía de las lecturas, las presentaciones de 'escribe mientras puedas' y las clases teóricas. Me queda un reto mayor pues esta materia me hará salir de mi zona de confort tanto como alumno como docente (O, V., comunicación personal, Julio 2016).

En lo personal la clase sigue siendo muy dinámica, [...] La clase sale fuera de lo tradicional, de lo cotidiano, tedioso y repetitivo (C. S., comunicación personal, noviembre 2017). 
Los testimonios muestran cómo cada estudiante a través del trabajo que desarrolla el profesorado percibe cambios en su vida y reconoce lo que le permite alcanzar un mejor aprendizaje; por tanto, una práctica docente activa y que atiende las necesidades del estudiantado mejora el proceso de formación y la calidad de los aprendizajes. Sin embargo, no es suficiente generar procesos activos, para Skliar (2017) si algo se aprende es cuando "nos damos cuenta de ello y para darnos cuenta necesitamos tiempo, espacio, soledad" (p. 151), en este sentido, el diario constituye un recurso valioso para que estudiantes cuenten con ese tiempo, espacio y soledad.

\subsection{Trabajo colaborativo}

Para comenzar el análisis desprendido de esta categoría, se hace necesario precisar que el paradigma socioconstructivista afirma que el proceso de aprender es un proceso social. Al respecto, Aranda (2015) señala que la colaboración es fundamental en el proceso de aprendizaje, y que esta puede ser favorecida en "entornos alternativos centrados en el aprendiz, en los que las y los estudiantes pueden trabajar juntos y ayudarse mutuamente en parejas o en grupos" (Sección Aprendizaje Mediado y Situado, párr. 8). Como da cuenta el siguiente testimonio:

Mi reflexión es que hay que dialogar mucho para saber qué puede hacer cada uno en un equipo, pues si no escuchas, puedes ser impositivo y hacer sentir mal a los otros (S. V., comunicación personal, octubre 2017).

El hecho de que cada estudiante identifique las interacciones que se dan con sus compañeros y, las estrategias que les ayudan a incorporar nuevos elementos a su estructura cognitiva, le permite también elaborar el trabajo con otros, así como desarrollar habilidades, destrezas distintas a las alcanzadas de manera individual, pues no se desarrollan de la misma manera. Además, la interacción-colaboración propicia la creación de comunidades de aprendizaje, y contribuye a explorar la no linealidad de los entornos de aprendizaje Barba, Valdiviezo y Aguilar (2018).

En los diarios se encuentra que el estudiantado valora lo que escucha de sus compañeros al brindarle diferentes perspectivas y puntos de vista, así como la importancia del trabajo transdisciplinar:

Trabajar en equipo ha sido siempre de mi agrado por lo que realizar dinámicas hará más rica esta clase sumado a que son equipos interdisciplinarios podemos aprender más y sobre todo a tener diferentes perspectivas, la solución de un problema puede que no se soluciona desde tu área de estudio, por lo que abordar las problemáticas con la caja vacía, será siempre una buena manera de empezar el objeto de estudio. (V. M., comunicación personal, noviembre 2017).

Del mismo modo los diarios muestran un gran énfasis en que trabajar en equipo es divertido, sin dejar de lado las dificultades que ello conlleva, desde no estar acostumbrados a hacerlo, pasando por lo complicado que es abrir su pensamiento a otras perspectivas, hasta reconocer los problemas para compaginar tiempos y recursos. Las personas participantes también manifiestan la emoción de logro al desarrollar las habilidades necesarias para trabajar en equipo.

Llegar a un acuerdo con el equipo, participar con ideas y adoptar las ideas de otros para al final tener un resultado que cumpla con los objetivos de la tarea suena fácil, sin embargo toma tiempo y permite abrir nuestro pensamiento a otras perspectivas; que me reafirman la relevancia del trabajo comunitario, traducido siempre en el resultado final. ;Me encantó ver a mis compañeros disfrutar aprendiendo! (M. R., comunicación personal, noviembre 2016).

Por otro lado, las narrativas muestran que la ayuda que reciben de los pares es muy valorada, pues de estar en un estado de confusión, han logrado claridad al recibir la ayuda de su par.

Durante la clase pude escuchar de mis compañeros sus experiencias dentro de la sesión 1 a la cual no pude asistir, fue enriquecedor escuchar sus comentarios acerca de los temas vistos ya que comprendi fácilmente lo que yo no habia visto, fueron muy claros y concisos sobre las ideas proporcionados, gracias a ello pude ponerme al corriente rápido y sin problemas (A. L., comunicación personal, julio 2016). 
Otro aspecto valioso y fundamental es la manifestación de sus emociones y sentimientos ante el trabajo que ejecutan con otros en el aula, ya sea al presentar un trabajo, al manifestar cómo es que han llegado a ciertas conclusiones, o simplemente valorar la importancia de estar al lado de otros en el momento del aprendizaje: Esta materia nos cambió como grupo (S. E., comunicación personal, julio 2016).También para recuperar algo que se perdió por no estar presente o vencer esa resistencia inicial a trabajar al lado de otros. A parte de esto, lograr un mayor conocimiento de los demás, como lo expresa este testimonio: Además esta actividad me permitió conocer un poco más a mis compañeros, escuchar sus intereses, admirar su estilo y conocer su sensibilidad. (A. W., comunicación personal, julio 2016). Roselli (2016) indica que:

El conocimiento es definido como un proceso de negociación o construcción conjunta de significados, y esto vale para todo el proceso de enseñanza-aprendizaje. Aunque el peso del concepto está puesto en el reconocimiento del valor de la interacción cognitiva entre pares, el aprendizaje colaborativo involucra también al docente y, en general, a todo el contexto de la enseñanza. No se trata, pues, de la aplicación circunstancial de técnicas grupales, sino de promover el intercambio y la participación de todos en la construcción de una cognición compartida (p. 224)

\subsection{Aprender jugando}

En varios diarios se identificaron testimonios que aluden al aprendizaje y la diversión. Para estos participantes la diversión constituye una dimensión que contribuye al aprendizaje; un testimonio señala:

Tengo el referente de que este dia la clase fue genial, muy productiva y divertida, el aprendizaje mediante el juego hace que el tedio desaparezca (A. G., comunicación personal, julio 2016).

En este proceso el juego toma un lugar de motivador, aligera procesos que podrían resultar estresantes o agobiantes.

Para Sicart (2014), la acción de jugar es apropiativa, es decir, permite que el sujeto se adueñe de la actividad y del contexto en el que esta tiene lugar. En este sentido, el juego le ofrece al estudiantado la oportunidad de asumir el protagonismo del proceso, más allá de que haya una consigna predefinida por el profesorado el juego facilita que estudiantes le den un sentido propio y lo signifiquen, tal como se observa en otro testimonio:

El desarrollo de la clase es bueno, me parece fascinante la forma de cómo [...] logra engranar los temas a través del juego y se encuentra un aprendizaje lúdico y significativo, con noción de causa, no cualquiera lo hace. (M. M., comunicación personal, noviembre 2017).

En la concepción de algunos participantes aprender jugando reporta buenos resultados, que están asociados a la capacidad de crear, pensar en otras formas y disponer de diferentes alternativas para dar soluciones a problemas habituales. De acuerdo con Bruner (1986) "el juego es un excelente medio de exploración que de por sí infunde estímulo" (p.79).

Me sentí motivada a aprender porque creo que será jugando aprendes y esta es una de las técnicas que mejores resultados dan (J, V., comunicación personal, noviembre 2017).

Aprender jugando también está asociado a un placer; al jugar se despliega un gozo que puede devenir en sentirse libre para imaginar y desplegar la curiosidad. Al respecto un testimonio señala:

La clase fue muy divertida y bonita. Primero por la actividad que hicimos de ir completando las figuras que habia en una hoja. En ella todos teniamos que dibujar lo primero que se nos ocurriera y usar nuestra imaginación. Noté que mientras todos dibujábamos, nuestra curiosidad hacia que volteáramos a ver las hojas de los demás para ver qué era lo que estaban haciendo (S. M., comunicación personal, diciembre 2016).

Cabe observar la relación que existe entre esta sensación de gozo y la disposición de las y los participantes al aprendizaje. En un testimonio se destaca: Son este tipo de actividades las que me hacen llegar emocionada a mi 
clase cada día porque siempre aprendo algo nuevo de forma divertida (P. D., comunicación personal, noviembre 2016).

Finalmente, en los diarios también se expresa que el estudiantado además de divertirse aprende, reafirma conceptos y convive con sus compañeros. El juego en estos testimonios no se limita a una práctica lúdica también remite a una experiencia que favorece la imaginación y la flexibilidad en los procesos de aprendizaje.

\section{CONCLUSIONES Y RECOMENDACIONES}

La investigación alcanzó su objetivo al fundamentar como la experiencia del uso de diario aporta beneficios tanto al estudiantado como al profesorado. Se observó que unas de las principales habilidades desarrolladas por estudiantes en su proceso de aprendizaje es la habilidad de reflexionar sobre su propia manera de pensar, analizar, criticar, evaluar y ofrecer argumentos que sustenten su pensamiento crítico y creativo, todo ello relacionado con la metacognición. Autores como Gamboa, Huegun y Jauregui (2015) mencionan que "Todo ese bagaje experiencial del alumno, a veces, está sin trabajar, sin articular ni explicitar. Es decir, está sin transferirlo al campo simbólico, pero eso no quiere decir que carezca de significado” (p. 77) por lo que el diario colabora a ese proceso de darle significado a la experiencia educativa, al proceso de aprender. Al escribir el diario, cada estudiante elabora un proceso de dotar significado a sus experiencias. Además de propiciar y fomentar una de las tareas fundamentales de la universidad que es la escritura académica (González, 2018).

El diario a diferencia de otras herramientas, como los exámenes, por ejemplo, le permite al estudiante darse cuenta de sus logros, de lo que le falta aprender, de lo que aún no le queda claro, de lo que facilitó su aprendizaje y cuál fue su actitud y disposición ante la novedad que conlleva la construcción del conocimiento. Lo anterior se incrementa al recibir la retroalimentación docente. De esta manera, el alumnado entra en un ambiente que le facilita el desarrollo de habilidades de orden superior donde la retroalimentación profunda, crítica y constructiva es fundamental.

El empleo del diario dentro de un planteamiento educativo crítico y constructivo y la utilización de técnicas de enseñanza activas, propicia el ajuste continuo del y la docente y estudiantes, iniciando un proceso continuo de flexibilidad y reajuste que deriva en una innovación permanente. En el entendido que innovar es combinar cosas para darle nuevas utilidades y mejorar los procesos.

Por otro lado, se advierten varias precauciones en el uso del diario:

Se requiere que el profesor o profesora no solo reciba el diario como parte del trabajo del alumno o alumna, sino que a través del proceso de retroalimentación se establezca una verdadera comunicación entre ambos y que no solo se considere como una actividad dada al estudiante. La retroalimentación deberá ser empleada por el y la docente para verificar si cada estudiante muestra cambios y toma en cuentas las orientaciones y sugerencias hechas ya sea verificando en las sesiones de clase o en lo que describe en las siguientes participaciones al diario.

Las indicaciones para elaborar el diario deben ser claras para que su escritura esté orientada a la experiencia de aprender. Por lo tanto, se requiere de consignas precisas, intencionadas y vinculadas al propósito del curso. Se debe buscar un nivel de profundización para que el alumnado vaya más allá de repetir lo que pasó en clase, ahonden en su aprendizaje y habilidades de orden superior, en la que también hagan visibles sus emociones y tensiones.

Como lo sugieren Barba, González y Barba (2014), el uso del diario se torna en un "factor clave para la mejora educativa" (p. 59) al poner de manifiesto lo que el y la docente ha hecho o ha dejado de hacer. Del mismo modo, da cuenta de lo que ha quedado aprendido y de lo que aún no fue asimilado por las y los estudiantes. Lo cual puede conducir a un proceso de innovación puesto que responde a las metodologías centradas en el aprendiz dónde este es el protagonista de su proceso de aprendizaje. Todo esto da pie a desarrollar la capacidad de autorregulación, autoevaluación, habilidades necesarias para desplegar su protagonismo. 
Del mismo modo, el uso del diario permitió enfatizar que es necesario trabajar a la par, el desarrollo de habilidades emocionales con habilidades cognitivas para procurar un desarrollo integral, porque es común separar ambas dimensiones. Esto último tiene que ver con la innovación, puesto que los nuevos conocimientos de la neurociencia están siendo aplicados a la educación, lo cual da luces para el diseño de ambientes de aprendizaje en donde se puedan incorporar dichos avances en favor de la mejora de la práctica docente y el proceso de aprendizaje del estudiantado. Los constantes cambios de un mundo globalizado, tecnificado y con grandes cantidades de información demanda estudiantes capaces de desenvolverse en las exigencias del entorno, mismo que "los impulsa a hacerse cargo de su aprendizaje y a ser cada vez menos dependientes del docente" (González, et al., 2017, p.77), lo que sin duda repercute de manera directa en la forma de llevar a cabo la enseñanza y el aprendizaje; implica efectuar procesos educativos dinámicos y activos, tal como lo mostraron los testimonios de las y los estudiantes a lo largo del análisis presentado.

En un examen, no exhaustivo, pero no menos importante, a través de la herramienta que brinda el Atlas Ti para hacer correlaciones entre los códigos de información se confirmó lo que ya se venía encontrando en el análisis individual de cada categoría: las correspondencias entre códigos. De tal manera que, por ejemplo, en relación al Trabajo docente se encuentra relacionado con Aprender jugando. Metacognición y Emociones. Y la categoría de Trabajo colaborativo, mantiene correlaciones con Metacognición y Trabajo docente. Lo que da cuenta de la multidiminencionalidad que se experimenta en los procesos educativos.

Se invita a continuar con estudios sobre el tema que permitan ampliar la mirada sobre este tipo de escritura en procesos educativos y de trabajo académico. También se recomienda que estudios posteriores en el tema, consideren ampliar la muestra e incluir otros recursos como proponen Canabal y Margalef (2017); lo que permitiría innovar el proceso de enseñanza, aprendizaje y evaluación, así como conocer cuáles inciden mejor en el aprendizaje. Además, construir fundamentos para la incorporación de metodologías activas, como la que subyace en esta investigación. Lo que representa líneas de investigación futuras, en las que además deberá considerarse, entre otros temas, cómo a través del diario se puede reorientar contenidos, motivar la autogestión del aprendizaje o la autoevaluación.

El diario es un recurso de doble beneficio, pues además de permitir al estudiante darse cuenta de aspectos fundamentales de su proceso de aprendizaje, al docente le facilita la evaluación y le permite mejorar e innovar en su práctica docente. Con esta experiencia interesa motivar a otros docentes a hacer uso de este recurso.

En términos generales, la experiencia en el uso del diario ha resultado muy enriquecedora y significativa; ha puesto luz sobre cuestiones subjetivas de las y los estudiantes que quedan de lado en la mayoría de los instrumentos empleados por los docentes porque recuperan su voz; pero también porque ha constituido una oportunidad de aquilatar el verdadero sentido de la formación docente, del trabajo y la responsabilidad que se tiene como profesores (Salgado, 2017).

Se espera que la presente investigación interese y anime a docentes a usar y explorar los beneficios que tiene el diario del y la estudiante para diseñar y llevar a cabo procesos de enseñanza y aprendizaje que vayan más allá de la transmisión y acumulación de información. Porque como dice Mora (2013) “¿Qué es lo que se salva del día? Los momentos de aprendizaje que se reviven y revisan conscientemente, aquello que recobras como 'bien vivido”' (p. 231). Debe salvarse día a día la tarea docente, el aporte a la sociedad.

\section{REFERENCIAS}

Aranda, L. (2015). Un acercamiento al aprendizaje colaborativo en educación superior. El aprendizaje colaborativo al alcance de todo. UAM - Azcapotzalco, UNAM. Lingüistica Aplicada. Recuperado de http://relinguistica.azc.u am.mx/no016/RL016_A03.htm

Ausubel, D.P. (2002). Adquisición y retención del conocimiento. Una perspectiva cognitiva. Barcelona: Paidós.

Báez y Otero (2013). La reflexión sobre el aprendizaje en el aula: El diario de Clase. En Xornada de Innovación Educativa. (pp.95-106). España: Universidad de Vigo. España. 
Barba, J.J., González, G. y Barba, R.A. (2014). Uso de los diarios del profesorado como instrumento de reflexión-sobrela-acción. Revista Española de Educación Física y Deportes, 405, 55-63.

Barba, L.R., Valdiviezo Aguilar, J. (2018). Gestión emergente de espacios colaborativos de aprendizaje. Revista Ibérica de Sistemas e Tecnologias de Información, 6 (1), 271-281. http://doi.org/10.17013/risti.n.pi-pf

Bruner, J. (1986). Juego, pensamiento y lenguaje. Perspectivas, 16 (1), 77-83.

Canabal, C. y Margalef, L. (2017). La retroalimentación: La clave para una evaluación orientada al aprendizaje. Profesorado. Revista de curriculum y formación del profesorado, 21(2), 149-170. Recuperado de http://www.red alyc.org/html/567/56752038009/

Carlino, P. (2003). La experiencia de escribir una tesis: contextos que la vuelven más difícil. Anales del Instituto de Lingüistica, 24, 41-62. Recuperado de https://bit.ly/2XBknpU

Cebrián, D., Pérez, R. y Cebrián, M. (2017). Estudio de la comunicación en la evaluación de los diarios de prácticas que favorecen la argumentación. Revista Prácticum, 2(1), 1-21. Recuperado de https://idp.gteavirtual.org/ojs/ index.php/iop/article/view/19

Cerchiaro, E., Paba, C. y Sánchez, L. (2011). Metacognición y Comprensión Lectora: una relación posible e intencional. DUAZARY. Revista de la Facultad de Ciencias de la Salud. 8(1), 99-111. Recuperado de https:// dialnet.unirioja.es/descarga/articulo/4788224.pdf

Delors, J. (1996). La educación encierra un tesoro. París: UNESCO.

Didi-Huberman, G. (2013). Cuando las imágenes toman posición. España: A. Machado

Elizondo, E., Rodríguez, J.V. y Rodríguez, I. (2018). La importancia de la emoción en el aprendizaje: Propuestas para mejorar la motivación de los estudiantes. Cuaderno de Pedagogía Universitaria, 15 (29), 3-11. Recuperado de h ttp://cuaderno.pucmm.edu.do/index.php/cuadernodepedagogia/article/view/296

Gamboa, E., Huegun, A. y Jauregui, P. (2015). La creación de conocimiento significativo desde la experiencia propia del alumnado: la reflexión sentida. Procedia - Social and Behavioral Sciences, 196, 75-79. https://doi.org/10.10 16/j.sbspro.2015.07.014

García, C. (2006). Habilidades de pensamiento de orden superior, epistemología y evaluación en el aula de clase. Lúmina, 7, 8-26. Recuperado de revistasum.umanizales.edu.co/ojs/index.php/Lumina/article/ download/1173/1249/

García, L. (2018). En modo selfie: reflexiones sobre la potencia de las selfies. Nómadas, 49, 103-119. http://doi.org/ $10.30578 /$ nomadas.n49a6

Gay, L. R. y Airasian, P. (1996). Educational Research. Competencies for Analysis y Application. Estados Unidos de Norteamérica: Prentice Hall.

Giraldo, C. (2015). La escritura en el aula como instrumento de aprendizaje. Estudio en universidades. Anfora, 22 (38), 39-58. Recuperado de: https://dialnet.unirioja.es/descarga/articulo/5151538.pdf

Goleman, D. (1997). Inteligencia emocional. Barcelona: Kairós.

González, N. (2018). El diario como estrategia didáctica para el desarrollo de la escritura académica en los estudiantes de la Educación Superior. Revista Cientifica ECOCIENCIA, 5, 1-20. Recuperado de http:// ecociencia.ecotec.edu.ec/upload/php/files/diciembreespe/12.pdf

González, L.A., Cárdenas, L.A. y Arellano, J.C. (2017). Desarrollo de habilidades del pensamiento de orden superior a través de actividades de desempeño. Revista Electrónica ANFEI Digital, 3(6), 1-9. Recuperado de http:// www.anfei.org.mx/revista/index.php/revista/article/view/360/1002

González, Y., Vargas, M.L., Gómez, M.I. y Méndez, A.M. (2017). Estrategias que favorecen el aprendizaje autónomo en estudiantes universitarios. Calidescopio, 73, 75 - 90. https://doi.org/10.33064/37crscsh903

Hortigüela, D. (2017). El pensamiento reflexivo y la adquisición de competencias docentes en educación física a través del empleo de técnicas narrativas. Revista Infancia, Educación y Aprendizaje, 3(2), 577-582. Recuperado de http://micologia.uv.cl/index.php/IEYA/article/view/783/762 
Jiménez, J.A., Rossi, F. y Gaitán, C. (2017). La Práctica reflexiva como posibilidad de construcción de saberes. Aportes a la formación docente en educación superior. Movimiento, 23 (2), 587-600. http://doi.org/10.22370/ieya.20 17.3.2.783

Jurado, D. (2011). El diario como un instrumento de autoformación e investigación. Revista Curriculum, 24, 173-200.

Larrosa; J. (2018). P de Profesor. Buenos Aires: Noveduc. Recuperado de http://ieie.udistrital.edu.co/pdf/Una \%20invitaci\%C3\%B3n\%20a\%20la\%20escritura.pdf

López, E. (2005). La educación emocional. Revista Interuniversitaria de Formación del Profesorado, 19(3), 153- 167. Recuperado de https://dialnet.unirioja.es/servlet/articulo?codigo $=2126770$

López, G y Roger, S. (2014). El diario del profesor como herramienta de evaluación cualitativa de un programa para aprender a pensar. Talincrea, 1(1), 3-26.

López, J.C.y Pérez, I. (2018) ¿Por qué es necesario una didáctica específica para la educación superior? Revista Científica ECOCIENCIA, 5(1), 1-17. Recuperado de http://ecociencia.ecotec.edu.ec/upload/php/files/febrero18/04.pdf

Martínez, M. (2013). Epistemología y Metodología Cualitativa en las Ciencias Sociales. México: Ed. Trillas.

Mayer, J. D., Caruso, D. y Salovey, P. (1999). Emotional intelligence meets traditional standards for an intelligence. Intelligence, 27, 267-298. Recuperado de https://bit.ly/36FfH6q

Messina, G. (2019). El oficio docente. Desde el ataque de nervios al deseo de saber. Con-Ciencia Educativa, 0, 49-56.

Monsalve, A. y Pérez. E. (2012). El diario pedagógico como una herramienta de investigación. Itinerario Educativo, 26(60), 117-128. Recuperado de https://dialnet.unirioja.es/descarga/articulo/5163235.pdf

Mora, Z. (2013). Escribir para sanar. Manual de ontoescritura. Amate Editorial. México.

Moreira, M.A. (2012). ¿Al final, qué es el aprendizaje significativo? Revista Qurriculum, 25, 29-56. Recuperado de h ttps://bit.ly/38NN7S9

Ojeda, F. (2017) Diarios íntimos de autores. La escritura de la vida. Culto. Recuperado de: http://culto.latercera.com /2017/12/15/diarios-intimos-autores-la-escritura-la-vida/

Ospina, D. (2016). El diario como estrategia didáctica. Recuperado de https://bit.ly/2PVfc1h

Paniagua, M. y Palos, U. (Coord.), (2009). Narrativas docentes en contextos innovadores. Guadalajara: ITESO.

Parga, M. (Julio y agosto 2007). Pensamiento de orden superior en diseño: Aportes del enfoque congnitivo a los procesos de formación de competencias para diseñar. En O. Echavarría. (Presidencia). II Encuentro Latinoamericano de Diseño: Diseño en Palermo. Universidad de Palermo, Buenos Aires, Argentina. Recuperado de https://bit.ly/2tuIBYL

Porlán, R. y Martín, J. (2000). El diario del profesor. Un recurso para investigación en el aula. España: Diada Editora S.L.

Rodríguez, L. (2014). Metodologías de enseñanza para un aprendizaje significativo de la histología. Revista Digital Universitaria, 15 (11), 1-16.

Roig, R. y Cobos, J.C. (2019). La Didáctica como elemento determinante en los nuevos escenarios de la Educación Superior. Revista Internacional d'Humanitats, 46-47, 129-142 Recuperado de http://www.hottopos.com/rih4 6/129-142Rosabel.pdf

Roselli, N. (2016). El aprendizaje colaborativo: Bases teóricas y estrategias aplicables en la enseñanza universitaria. Propósitos y Representaciones, 4(1), 219-280. http://doi.org/10.20511/pyr2016.v4n1.90

Salgado, A. (Noviembre, 2017). Mirando al "server" la Investigación Cualitativa. Innovación para la formación Integral. Experiencias Innovadoras. En S. Rubín. (Presidencia). Innovación para la Formación Integral: Experiencias Educativas Innovadoras Universidad Popular Autónoma del Estado de Puebla, México.

Sánchez, I. (2015). La andragogía de Malcom Knowles: Teoría y Tecnología de la educación de adultos. Recuperado de https://bit.ly/295w616

Sicart, M. (2014) Play Matters. Massachusetts: MIT Press

Sicilia, A. (1999). El diario del alumnado como técnica de investigación en Educación Física. Educación Física y Deportes, (58), 25-33. Recuperado de https://bit.ly/2y7CMPZ

Skliar, C. (2017). Pedagogias de las diferencias. Notas, fragmentos, incertidumbres. Buenos Aires: Noveduc 
Alma Salgado Ramírez, et al. La experiencia del estudiantado mediante el uso del diario. ¿UNa ESTR...

Vain, D. (2003). El diario académico: una estrategia para la formación de docentes reflexivos. Perfiles educativos, 25(100), 56-68. Recuperado de https://bit.ly/35z4RyD

\section{Notas}

1 Da cuenta de un antes y un después

\section{BY-NC-ND}

